\title{
Quantum Walk in Terms of Quantum Bernoulli Noise and Quantum Central Limit Theorem for Quantum Bernoulli Noise
}

\author{
Caishi Wang $\mathbb{D}^{1},{ }^{1}$ Ce Wang $\left(\mathbb{D},{ }^{2}\right.$ Yuling Tang $\left(\mathbb{D},{ }^{1}\right.$ and Suling Ren ${ }^{1}{ }^{1}$ \\ ${ }^{1}$ School of Mathematics and Statistics, Northwest Normal University, Lanzhou, Gansu 730070, China \\ ${ }^{2}$ School of Mathematics and Statistics, Wuhan University, Wuhan, Hubei 430072, China \\ Correspondence should be addressed to Caishi Wang; wangcs@nwnu.edu.cn
}

Received 24 August 2017; Revised 10 December 2017; Accepted 28 December 2017; Published 24 January 2018

Academic Editor: Antonio Scarfone

Copyright (C) 2018 Caishi Wang et al. This is an open access article distributed under the Creative Commons Attribution License, which permits unrestricted use, distribution, and reproduction in any medium, provided the original work is properly cited.

\begin{abstract}
As a unitary quantum walk with infinitely many internal degrees of freedom, the quantum walk in terms of quantum Bernoulli noise (recently introduced by Wang and Ye) shows a rather classical asymptotic behavior, which is quite different from the case of the usual quantum walks with a finite number of internal degrees of freedom. In this paper, we further examine the structure of the walk. By using the Fourier transform on the state space of the walk, we obtain a formula that links the moments of the walk's probability distributions directly with annihilation and creation operators on Bernoulli functionals. We also prove some other results on the structure of the walk. Finally, as an application of these results, we establish a quantum central limit theorem for the annihilation and creation operators themselves.
\end{abstract}

\section{Introduction}

Quantum walks are quantum analogs of the classical random walk, which have found wide application in quantum information, quantum computing, and many other fields [13]. In the past fifteen years, quantum walks with a finite number of internal degrees of freedom have been intensively studied and many deep results have been obtained of them (see, e.g., [2, 4-9] and references therein). One typical result in this aspect is the finding that those walks have quite different asymptotic behavior, compared to their classical counterparts. For example, Konno [10] proved that, for localized initial states, a discrete-time quantum walk on the line with a finite number of internal degrees of freedom usually has a limit distribution with scaling speed $n$, which is far from being Gaussian. Similar properties have also been found for continuous-time quantum walks with a finite number of internal degrees of freedom [11]. However, little attention has been paid to quantum walks with infinitely many internal degrees of freedom, which are of interest at least from a theoretical point of view.

Quantum Bernoulli noise is the family of annihilation and creation operators acting on Bernoulli functionals, which satisfies a canonical anticommutation relation (CAR) in equal time, and can be viewed as a discrete-time counterpart of the quantum white noise introduced by Huang [12]. Recently, with the help of quantum Bernoulli noise, Wang and Ye [13] have constructed a discrete-time quantum walk with infinitely many internal degrees of freedom, which we call the QBN-based walk below.

The QBN-based walk takes $l^{2}(\mathbb{Z}, \mathscr{H})$ as its state space, where $\mathscr{H}$ denotes the space of square integrable Bernoulli functionals, which is infinitely dimensional. It has been shown [13] that, for some localized initial states, the QBNbased walk has a Gaussian limit distribution with scaling speed $\sqrt{n}$, which is in striking contrast with the case of the usual discrete-time quantum walks with a finite number of internal degrees of freedom. Machida [6] has found that, for a very particular nonlocalized initial state, a discrete-time quantum walk on the line with 2 internal degrees of freedom can generate a Gaussian limit distribution with scaling speed $n$. And he wondered [6] whether a discrete-time quantum walk can generate a Gaussian limit distribution with scaling speed $\sqrt{n}$. The QBN-based walk then seems to give an answer to this question in a way.

In this paper, we would like to further examine the structure property of the QBN-based walk and show its 
application to quantum probability. Our main work is as follows.

Let $\left\{\partial_{k}, \partial_{k}^{*}\right\}_{k \geq 0}$ be quantum Bernoulli noise, namely, annihilation and creation operators on $\mathscr{H}$, and $X$ be the position operator in $l^{2}(\mathbb{Z}, \mathscr{H})$. In the first part of the present paper, by using the Fourier transform on $l^{2}(\mathbb{Z}, \mathscr{H})$, we obtain a representation of the QBN-based walk in the momentum space. In particular, we obtain the following relations:

$$
\begin{array}{r}
\left\langle\Phi_{n}, X^{r} \Phi_{n}\right\rangle_{l^{2}(\mathbb{Z}, \mathscr{H})}=\left\langle\varphi,\left[\sum_{k=0}^{n-1}\left(\partial_{k}^{*}+\partial_{k}\right)\right]^{r} \varphi\right\rangle_{\mathscr{H}}, \\
r \geq 1,
\end{array}
$$

where $\Phi_{n} \in l^{2}(\mathbb{Z}, \mathscr{H})$ denotes the state of the walk at time $n \geq 0$ and $\varphi=\Phi_{0}(0)$. Since the quantity $\left\langle\Phi_{n}, X^{r} \Phi_{n}\right\rangle_{l^{2}(\mathbb{Z}, \mathscr{H})}$ is exactly the $r$ th moment of the walk's probability distribution at time $n \geq 0$, the above relations actually provide a formula that links the moments of the walk's probability distributions directly with the annihilation and creation operators.

Quantum central limit theorems are quantum analogs of the classical central limit theorem, which deal with observables from a quantum probability point of view. Cushen and Hudson [14] established the quantum central limit theorem for a pair of conjugate observables $P$ and $Q$ (i.e., such that $[P, Q]=i I)$, which was later generalized to arbitrary CCR algebras by Quaegebeur [15]. Giri and von Waldenfels [16] proved an algebraic quantum central limit theorem in the setting of $*$-algebra by using the method of noncommutative moments. Voiculescu has developed a noncommutative probability theory (now known as the free probability theory), which offers the free central limit theorem associated with the free independence [17]. There are many other types of quantum central limit theorems in the literature (see, e.g., [18-22] and references therein).

Obviously, operators $\partial_{k}^{*}+\partial_{k}, k \geq 0$, are observables on $\mathscr{H}$, which serves as the coin space of the QBN-based walk. In fact, these operators just play the role of quantum bias in the construction of the QBN-based walk. In the second part of the present paper, as application of our results mentioned above, we prove a quantum central limit theorem for observables $\partial_{k}^{*}+\partial_{k}, k \geq 0$.

The paper is organized as follows. In Section 2, we briefly recall main notions and facts about quantum Bernoulli noise. Section 3 describes the quantum walk introduced by Wang and Ye [13], namely, the QBN-based walk. Our main work then lies in Sections 4 and 5. Finally in Section 6, we make some conclusion remarks.

Notation and Conventions. Throughout, $\mathbb{Z}$ always denotes the set of all integers, while $\mathbb{N}$ means the set of all nonnegative integers. We denote by $\Gamma$ the finite power set of $\mathbb{N}$; namely,

$$
\Gamma=\{\sigma \mid \sigma \subset \mathbb{N}, \# \sigma<\infty\}
$$

where $\# \sigma$ means the cardinality of $\sigma$. Unless otherwise stated, letters like $j, k$, and $n$ stand for nonnegative integers, namely, elements of $\mathbb{N}$.

\section{Quantum Bernoulli Noise}

In this section, we briefly recall main notions and facts about quantum Bernoulli noise. We refer to $[13,23,24]$ for details.

Let $\Omega=\{-1,1\}^{\mathbb{N}}$ be the set of all mappings $\omega: \mathbb{N} \mapsto$ $\{-1,1\}$ and $\left(\zeta_{n}\right)_{n \geq 0}$ the sequence of canonical projections on $\Omega$ given by

$$
\zeta_{n}(\omega)=\omega(n), \quad \omega \in \Omega .
$$

Let $\mathscr{F}$ be the $\sigma$-field on $\Omega$ generated by the sequence $\left(\zeta_{n}\right)_{n \geq 0}$ and $\left(p_{n}\right)_{n \geq 0}$ a given sequence of positive numbers with the property that $0<p_{n}<1$ for all $n \geq 0$. It is known [25] that there exists a unique probability measure $\mathbb{P}$ on $\mathscr{F}$ such that

$$
\begin{aligned}
\mathbb{P} \circ & \left(\zeta_{n_{1}}, \zeta_{n_{2}}, \ldots, \zeta_{n_{k}}\right)^{-1}\left\{\left(\epsilon_{1}, \epsilon_{2}, \ldots, \epsilon_{k}\right)\right\} \\
= & \prod_{j=1}^{k} p_{j}^{\left(1+\epsilon_{j}\right) / 2}\left(1-p_{j}\right)^{\left(1-\epsilon_{j}\right) / 2}
\end{aligned}
$$

for $n_{j} \in \mathbb{N}, \epsilon_{j} \in\{-1,1\}(1 \leq j \leq k)$ with $n_{i} \neq n_{j}$ when $i \neq j$ and $k \in \mathbb{N}$ with $k \geq 1$. Thus one has a probability measure space $(\Omega, \mathscr{F}, \mathbb{P})$, which is referred to as the Bernoulli space and random variables on it are known as Bernoulli functionals.

Let $Z=\left(Z_{n}\right)_{n \geq 0}$ be the sequence of Bernoulli functionals defined by

$$
Z_{n}=\frac{\zeta_{n}+q_{n}-p_{n}}{2 \sqrt{p_{n} q_{n}}}, \quad n \geq 0,
$$

where $q_{n}=1-p_{n}$. Clearly $Z=\left(Z_{n}\right)_{n \geq 0}$ is an independent sequence of random variables on the probability measure $\operatorname{space}(\Omega, \mathscr{F}, \mathbb{P})$.

Let $\mathscr{H}$ be the space of square integrable complex-valued Bernoulli functionals; namely,

$$
\mathscr{H}=L^{2}(\Omega, \mathscr{F}, \mathbb{P}) .
$$

We denote by $\langle\cdot, \cdot\rangle$ the usual inner product of the space $\mathscr{H}$ and by $\|\cdot\|$ the corresponding norm. It is known [25] that $Z$ has the chaotic representation property. Thus $\mathscr{H}$ has $\left\{Z_{\sigma} \mid \sigma \in \Gamma\right\}$ as its orthonormal basis, where $Z_{\emptyset}=1$ and

$$
Z_{\sigma}=\prod_{j \in \sigma} Z_{j}, \quad \sigma \in \Gamma, \sigma \neq \emptyset,
$$

which shows that $\mathscr{H}$ is an infinite dimensional complex Hilbert space.

Lemma 1 (see [23]). For each $k \in \mathbb{N}$, there exists a bounded operator $\partial_{k}$ on $\mathscr{H}$ such that

$$
\partial_{k} Z_{\sigma}=\mathbf{1}_{\sigma}(k) Z_{\sigma \backslash k}, \quad \sigma \in \Gamma,
$$

where $\sigma \backslash k=\sigma \backslash\{k\}$ and $\mathbf{1}_{\sigma}(k)$ is the indicator of $\sigma$ as a subset of $\mathbb{N}$.

Lemma 2 (see [23]). Let $k \in \mathbb{N}$. Then $\partial_{k}^{*}$, the adjoint of operator $\partial_{k}$ has the following property:

$$
\partial_{k}^{*} Z_{\sigma}=\left[1-\mathbf{1}_{\sigma}(k)\right] Z_{\sigma \cup k} \quad \sigma \in \Gamma,
$$

where $\sigma \cup k=\sigma \cup\{k\}$. 
The operator $\partial_{k}$ and its adjoint $\partial_{k}^{*}$ are usually known as the annihilation and creation operators acting on Bernoulli functionals, respectively.

Definition 3 (see [23]). The family $\left\{\partial_{k}, \partial_{k}^{*}\right\}_{k \geq 0}$ of annihilation and creation operators is called quantum Bernoulli noise.

The next lemma shows that quantum Bernoulli noise satisfies the canonical anticommutation relations (CAR) in equal time.

Lemma 4 (see [23]). Let $k, l \in \mathbb{N}$. Then it holds true that

$$
\begin{aligned}
\partial_{k} \partial_{l} & =\partial_{l} \partial_{k}, \\
\partial_{k}^{*} \partial_{l}^{*} & =\partial_{l}^{*} \partial_{k}^{*}, \\
\partial_{k}^{*} \partial_{l} & =\partial_{l} \partial_{k}^{*}, \quad(k \neq l), \\
\partial_{k} \partial_{k} & =\partial_{k}^{*} \partial_{k}^{*}=0, \\
\partial_{k} \partial_{k}^{*}+\partial_{k}^{*} \partial_{k} & =I,
\end{aligned}
$$

where I is the identity operator on $\mathscr{H}$.

Lemma 5 (see [13]). For $n \geq 0$, write $R_{n}=(1 / 2)\left(\partial_{n}^{*}+\partial_{n}+I\right)$ and $L_{n}=(1 / 2)\left(\partial_{n}^{*}+\partial_{n}-I\right)$. Then both $R_{n}$ and $L_{n}$ are selfadjoint operators on $\mathscr{H}$, and moreover

$$
\begin{aligned}
R_{n}^{2} & =R_{n}, \\
R_{n} L_{n} & =L_{n} R_{n}=0, \\
L_{n}^{2} & =-L_{n} .
\end{aligned}
$$

It follows easily from Lemma 4 that operators $R_{n}, L_{n}, n \geq$ 0 , form a commutative family; namely,

$$
\begin{aligned}
& R_{k} R_{l}=R_{l} R_{k}, \\
& R_{k} L_{l}=L_{l} R_{k}, \\
& L_{k} L_{l}=L_{l} L_{k}
\end{aligned}
$$

hold for all $k, l \geq 0$.

\section{QBN-Based Walk}

The present section describes the quantum walk introduced in [13], namely, the QBN-based walk mentioned above.

Recall that $\mathscr{H}=L^{2}(\Omega, \mathscr{F}, \mathbb{P})$, the space of square integrable complex-valued Bernoulli functionals. Let $l^{2}(\mathbb{Z}, \mathscr{H})$ be the space of square summable functions defined on $\mathbb{Z}$ and valued in $\mathscr{H}$; namely,

$$
l^{2}(\mathbb{Z}, \mathscr{H})=\left\{\Phi: \mathbb{Z} \longrightarrow \mathscr{H} \mid \sum_{x=-\infty}^{\infty}\|\Phi(x)\|^{2}<\infty\right\} .
$$

Then $l^{2}(\mathbb{Z}, \mathscr{H})$ remains a complex Hilbert space, whose inner product $\langle\cdot, \cdot\rangle_{l^{2}}$ is given by

$$
\langle\Phi, \Psi\rangle_{l^{2}}=\sum_{x=-\infty}^{\infty}\langle\Phi(x), \Psi(x)\rangle, \quad \Phi, \Psi \in l^{2}(\mathbb{Z}, \mathscr{H}),
$$

where $\langle\cdot, \cdot\rangle$ denotes the inner product of $\mathscr{H}$ as indicated in Section 2 . By convention, we denote by $\|\cdot\|_{l^{2}}$ the norm induced by $\langle\cdot, \cdot\rangle_{l}$. Note that $l^{2}(\mathbb{Z}, \mathscr{H})$ has a countable orthonormal basis $\left\{\phi_{z, \sigma} \mid z \in \mathbb{Z}, \sigma \in \Gamma\right\}$, where $\phi_{z, \sigma}: \mathbb{Z} \rightarrow \mathscr{H}$ is defined by

$$
\phi_{z, \sigma}(x)= \begin{cases}Z_{\sigma}, & x=z ; \\ 0, & x \neq z, x \in \mathbb{Z} .\end{cases}
$$

Thus $l^{2}(\mathbb{Z}, \mathscr{H})$ is separable.

As usual, a vector $\Phi \in l^{2}(\mathbb{Z}, \mathscr{H})$ is called a state if it satisfies the normalized condition $\|\Phi\|_{l^{2}}=1$.

Definition 6 (see [13]). The QBN-based walk is such a quantum walk whose state space is $l^{2}(\mathbb{Z}, \mathscr{H})$ and whose time evolution is governed by

$$
\begin{array}{r}
\Phi_{n+1}(x)=R_{n} \Phi_{n}(x-1)+L_{n} \Phi_{n}(x+1), \\
x \in \mathbb{Z}, n \geq 0,
\end{array}
$$

where $\Phi_{n} \in l^{2}(\mathbb{Z}, \mathscr{H})$ denotes the state of the walk at time $n \geq 0$.

Let $\left(\Phi_{n}\right)_{n \geq 0}$ be the state sequence of the QBN-based walk. Then the function $x \mapsto\left\|\Phi_{n}(x)\right\|^{2}$ makes a probability distribution on $\mathbb{Z}$, which is called the probability distribution of the walk at time $n \geq 0$. In particular, $\left\|\Phi_{n}(x)\right\|^{2}$ is the probability that the quantum walker is found at position $x \in$ $\mathbb{Z}$ at time $n \geq 0$. As usual, the QBN-based walk is assumed to start at position $x=0$, which implies that its initial state $\Phi_{0}$ satisfies $\left\|\Phi_{0}(0)\right\|^{2}=1$ and $\Phi(x)=0$ for $x \in \mathbb{Z}$ with $x \neq 0$.

Remark 7. It is well known that $l^{2}(\mathbb{Z}, \mathscr{H}) \cong l^{2}(\mathbb{Z}) \otimes \mathscr{H}$. Thus, $l^{2}(\mathbb{Z})$ describes the position of the QBN-based walk, while $\mathscr{H}$ describes the internal degrees of freedom of the walk. As shown in Section 2, the dimension of $\mathscr{H}$ is infinite, which means that the QBN-based walk has infinitely many internal degrees of freedom.

Lemma 8 (see [13]). For each $n \geq 0$, there exists a unitary operator $\mathscr{U}_{n}$ on $l^{2}(\mathbb{Z}, \mathscr{H})$ such that

$$
\begin{array}{r}
{\left[\mathcal{U}_{n} \Phi\right](x)=R_{n} \Phi(x-1)+L_{n} \Phi(x+1),} \\
x \in \mathbb{Z}, \Phi \in l^{2}(\mathbb{Z}, \mathscr{H}), \\
{\left[\mathcal{U}_{n}^{*} \Phi\right](x)=R_{n} \Phi(x+1)+L_{n} \Phi(x-1),} \\
x \in \mathbb{Z}, \Phi \in l^{2}(\mathbb{Z}, \mathscr{H}),
\end{array}
$$

where $\mathcal{U}_{n}^{*}$ denotes the adjoint of $\boldsymbol{u}_{n}$.

One can verify that unitary operators $\mathscr{U}_{n}, n \geq 0$, commute mutually; namely, $\mathscr{U}_{m} \mathscr{U}_{n}=\mathscr{U}_{n} \mathscr{U}_{m}$ for all $m, n \geq 0$. The next lemma shows that the QBN-based walk belongs to the category of unitary quantum walks. 
Lemma 9 (see [13]). The QBN-based walk has a unitary representation; more precisely,

$$
\Phi_{n+1}=\mathscr{U}_{n} \Phi_{n}=\left(\prod_{k=0}^{n} \mathcal{u}_{k}\right) \Phi_{0}, \quad n \geq 0,
$$

where $\Phi_{n}$ is the state of the walk at time $n \geq 0$.

\section{Structure Property of QBN-Based Walk}

In this section, we apply the Fourier transform theory to the QBN-based walk and examine its structure property. We continue to use the notation made in previous sections.

4.1. Fourier Transform on State Space. Consider $L^{2}([0,2 \pi], \mathscr{H})$, the space of all functions $f:[0,2 \pi] \rightarrow \mathscr{H}$ that are Bochner integrable [26] with respect to Lebesgue measure and satisfy condition $\int_{0}^{2 \pi}\|f(t)\|^{2} d t<\infty$. It is known that $L^{2}([0,2 \pi], \mathscr{H})$ is a Hilbert space with the inner product $\langle\cdot, \cdot\rangle_{L^{2}}$ given by

$$
\langle f, g\rangle_{L^{2}}=\frac{1}{2 \pi} \int_{0}^{2 \pi}\langle f(t), g(t)\rangle d t
$$

$$
f, g \in L^{2}([0,2 \pi], \mathscr{H}),
$$

where $\langle\cdot, \cdot\rangle$ denotes the inner product of $\mathscr{H}$ as indicated in Section 2.

A direct verification shows that the system $\left\{e_{z, \sigma} \mid z \in\right.$ $\mathbb{Z}, \sigma \in \Gamma\}$ is orthonormal in $L^{2}([0,2 \pi], \mathscr{H})$, where $e_{z, \sigma}$ : $[0,2 \pi] \rightarrow \mathscr{H}$ is defined by

$$
e_{z, \sigma}(t)=e^{i z t} Z_{\sigma}, \quad t \in[0,2 \pi] .
$$

We denote by $L^{2}(\mathbb{S}, \mathscr{H})$ the closed subspace of $L^{2}([0,2 \pi], \mathscr{H})$ spanned by the system $\left\{e_{z, \sigma} \mid z \in \mathbb{Z}, \sigma \in \Gamma\right\}$. Then $L^{2}(\mathbb{S}, \mathscr{H})$ together with $\langle\cdot, \cdot\rangle_{L^{2}}$ forms a separable complex Hilbert space.

Clearly $\left\{e_{z, \sigma} \mid z \in \mathbb{Z}, \sigma \in \Gamma\right\}$ is a countable orthonormal basis of $L^{2}(\mathbb{S}, \mathscr{H})$. This, together with the fact that the family $\left\{\phi_{z, \sigma} \mid z \in \mathbb{Z}, \sigma \in \Gamma\right\}$ is a countable orthonormal basis of $l^{2}(\mathbb{Z}, \mathscr{H})$, yields that there exists an isometric isomorphism $\mathrm{F}: l^{2}(\mathbb{Z}, \mathscr{H}) \rightarrow L^{2}(\mathbb{S}, \mathscr{H})$ such that

$$
\mathrm{F} \phi_{z, \sigma}=e_{z, \sigma}, \quad z \in \mathbb{Z}, \sigma \in \Gamma .
$$

The mapping $\mathrm{F}$ is then called the Fourier transform on $l^{2}(\mathbb{Z}, \mathscr{H})$.

It is easy to see that $\mathrm{F}^{-1}=\mathrm{F}^{*}$; namely, $\mathrm{F}$ is a unitary operator from $l^{2}(\mathbb{Z}, \mathscr{H})$ to $L^{2}(\mathbb{S}, \mathscr{H})$. Let $\Phi \in l^{2}(\mathbb{Z}, \mathscr{H})$ and $\widehat{\Phi}=\mathrm{F} \Phi$. Then one can prove that

$$
\lim _{m, n \rightarrow \infty} \int_{0}^{2 \pi}\left\|\widehat{\Phi}(t)-\sum_{x=-m}^{n} e^{i x t} \Phi(x)\right\|^{2} d t=0,
$$

which justifies the name of $F$. As usual, $F \Phi$ is called the Fourier transform of $\Phi$. It can also be proven that the inverse $\mathrm{F}^{-1}$ of $\mathrm{F}$ admits the following representation:

$$
\left[\mathrm{F}^{-1} f\right](x)=\frac{1}{2 \pi} \int_{0}^{2 \pi} e^{-i x t} f(t) d t
$$

$$
x \in \mathbb{Z}, f \in L^{2}(\mathbb{S}, \mathscr{H}),
$$

where the integral on the righthand side means the Bochner integral.

Just as in the scalar case, the position operator $X$ in $l^{2}(\mathbb{Z}, \mathscr{H})$ is defined by

$$
[X \Phi](x)=x \Phi(x), \quad x \in \mathbb{Z}, \Phi \in \operatorname{dom} X,
$$

where $\operatorname{dom} X$, the domain of $X$, is given by

$\operatorname{dom} X$

$$
=\left\{\left.\Phi \in l^{2}(\mathbb{Z}, \mathscr{H})\left|\sum_{x=-\infty}^{\infty}\right| x\right|^{2}\|\Phi(x)\|^{2}<\infty\right\} .
$$

It can be verified that $X$ is self-adjoint, and every integer $x \in$ $\mathbb{Z}$ is its eigenvalue with

$$
X \phi_{x, \sigma}=x \phi_{x, \sigma}, \quad \forall \sigma \in \Gamma .
$$

Let $r \geq 1$ be a positive integer. Then, by the theory of spectral resolution for self-adjoint operators [27], $X^{r}$ is well defined and remains a self-adjoint operator in $l^{2}(\mathbb{Z}, \mathscr{H})$, and moreover, its domain is determined by

$$
\begin{aligned}
& \operatorname{dom} X^{r} \\
& \quad=\left\{\left.\Phi \in l^{2}(\mathbb{Z}, \mathscr{H})\left|\sum_{x=-\infty}^{\infty}\right| x\right|^{2 r}\|\Phi(x)\|^{2}<\infty\right\}
\end{aligned}
$$

and its action is given by

$$
\left[X^{r} \Phi\right](x)=x^{r} \Phi(x), \quad x \in \mathbb{Z}, \Phi \in \operatorname{dom} X^{r} .
$$

Remark 10. Let $r \geq 1$ be a positive integer and $f:[0,2 \pi] \rightarrow$ $\mathscr{H}$ a continuous function that has continuous derivatives up to order $r$. Suppose that $f^{(j)} \in L^{2}(\mathbb{S}, \mathscr{H})$ for all $0 \leq j \leq r$. Then $\mathrm{F}^{-1} f \in \operatorname{dom} X^{r}$, and moreover

$$
\mathrm{F} X^{r} \mathrm{~F}^{-1} f=(-\mathrm{i})^{r} f^{(r)} .
$$

4.2. Structure Property. This subsection focuses on exploring the structure property of the QBN-based walk. Recall that the QBN-based walk takes $l^{2}(\mathbb{Z}, \mathscr{H})$ as its state space. Thus we may call $L^{2}(\mathbb{S}, \mathscr{H})$ the momentum space of the walk.

Theorem 11. Let $n \geq 0$ and $\mathscr{V}_{n}=\mathrm{F}_{\mathcal{U}_{n}} \mathrm{~F}^{-1}$. Then $\mathscr{V}_{n}$ is $a$ unitary operator on $L^{2}(\mathbb{S}, \mathscr{H})$, and moreover $\mathscr{V}_{n}$ admits the following representation:

$$
\left[\mathscr{V}_{n} f\right](t)=\left[e^{i t} R_{n}+e^{-i t} L_{n}\right] f(t) \text { a.e. in }[0,2 \pi],
$$

where $f \in L^{2}(\mathbb{S}, \mathscr{H})$. 
Proof. It is easy to verify that $\mathscr{V}_{n}$ is a unitary operator on $L^{2}(\mathbb{S}, \mathscr{H})$. Now define

$$
g(t)=\left[e^{i t} R_{n}+e^{-i t} L_{n}\right] f(t), \quad t \in[0,2 \pi] .
$$

Then $g \in L^{2}(\mathbb{S}, \mathscr{H})$. Thus, to prove (30), we need only to verify $\mathscr{V}_{n} f=g$.

Let $z \in \mathbb{Z}, \sigma \in \Gamma$. Then, by writing $\Phi=\mathrm{F}^{-1} f$, we have

$$
\begin{aligned}
\left\langle e_{z, \sigma}, \mathscr{V}_{n} f\right\rangle_{L^{2}} & =\left\langle\phi_{z, \sigma}, \mathscr{U}_{n} \mathrm{~F}^{-1} f\right\rangle_{l^{2}} \\
& =\left\langle Z_{\sigma},\left[\mathcal{U}_{n} \Phi\right](z)\right\rangle \\
& =\left\langle Z_{\sigma}, R_{n} \Phi(z-1)+L_{n} \Phi(z+1)\right\rangle .
\end{aligned}
$$

On the other hand, by using properties of the Bochner integrals as well as the representation of $\mathrm{F}^{-1}$, we can work out

$$
\begin{aligned}
\left\langle e_{z, \sigma}, g\right\rangle_{L^{2}} & \\
= & \frac{1}{2 \pi} \int_{0}^{2 \pi}\left\langle e^{i z t} Z_{\sigma},\left\{e^{i t} R_{n}+e^{-i t} L_{n}\right\} f(t)\right\rangle d t \\
= & \left\langle R_{n} Z_{\sigma}, \frac{1}{2 \pi} \int_{0}^{2 \pi} e^{-i(z-1) t} f(t) d t\right\rangle \\
& +\left\langle L_{n} Z_{\sigma}, \frac{1}{2 \pi} \int_{0}^{2 \pi} e^{-i(z+1) t} f(t) d t\right\rangle \\
= & \left\langle R_{n} Z_{\sigma},\left[\mathrm{F}^{-1} f\right](z-1)\right\rangle \\
& +\left\langle L_{n} Z_{\sigma},\left[\mathrm{F}^{-1} f\right](z+1)\right\rangle \\
= & \left\langle Z_{\sigma}, R_{n} \Phi(z-1)+L_{n} \Phi(z+1)\right\rangle .
\end{aligned}
$$

Thus

$$
\left\langle e_{z, \sigma}, \mathscr{V}_{n} f\right\rangle_{L^{2}}=\left\langle e_{z, \sigma}, g\right\rangle_{L^{2}}, \quad \forall z \in \mathbb{Z}, \forall \sigma \in \Gamma,
$$

which implies $\mathscr{V}_{n} f=g$.

Theorem 11 allows us to deal with the QBN-based walk in the momentum space $L^{2}(\mathbb{S}, \mathscr{H})$. In the following, we set

$$
\mathscr{V}_{n}=\mathrm{F}_{{ }_{n}} \mathrm{~F}^{-1}, \quad n \geq 0 .
$$

Clearly $\left\{\mathscr{V}_{n} \mid n \geq 0\right\}$ is a commutative family of unitary operators. The next theorem then offers a representation of the QBN-based walk in the momentum space $L^{2}(\mathbb{S}, \mathscr{H})$.

Theorem 12. Let $\left(\Phi_{n}\right)_{n \geq 0} \subset l^{2}(\mathbb{Z}, \mathscr{H})$ be the state sequence of the QBN-based walk, and $f_{n}=\mathrm{F} \Phi_{n}, n \geq 0$. Then $\left\|f_{0}\right\|_{L^{2}}=1$ and

$$
f_{n+1}=\mathscr{V}_{n} f_{n}=\left(\prod_{k=0}^{n} \mathscr{V}_{k}\right) f_{0}, \quad n \geq 0 .
$$

Proof. This is an immediate consequence of Lemma 9 and Theorem 11 together with properties of the Fourier transform F.
Remark 13. Let $n \geq 0$ and $f_{n}$ the same as in Theorem 12 . Then, on interval $[0,2 \pi]$, one has

$$
f_{n+1}(t)=\left[\prod_{k=0}^{n}\left(e^{i t} R_{k}+e^{-i t} L_{k}\right)\right] f_{0}(t) \text { a.e. }
$$

Recall that $X$ denotes the position operator in the state space $l^{2}(\mathbb{Z}, \mathscr{H})$ of the QBN-based walk. The next theorem then interprets the meaning of the quantity $\left\langle\Phi_{n}, X^{r} \Phi_{n}\right\rangle_{l^{2}}$.

Theorem 14. Let $\left(\Phi_{n}\right)_{n \geq 0} \subset l^{2}(\mathbb{Z}, \mathscr{H})$ be the state sequence of the $Q B N$-based walk, where the initial state $\Phi_{0}$ satisfies $\Phi_{0}(x)=0, x \neq 0, x \in \mathbb{Z}$. Then

$$
\Phi_{n} \in \operatorname{dom} X^{r}, \quad n \geq 0, r \geq 1,
$$

and moreover, for all $n \geq 0, r \geq 1$, the quantity $\left\langle\Phi_{n}, X^{r} \Phi_{n}\right\rangle_{l^{2}}$ is exactly the rth moment of the probability distribution of the walk at time n; namely,

$$
\left\langle\Phi_{n}, X^{r} \Phi_{n}\right\rangle_{l^{2}}=\sum_{x=-\infty}^{\infty} x^{r}\left\|\Phi_{n}(x)\right\|^{2} .
$$

Proof. Let $n \geq 0, r \geq 1$. Since $\Phi_{0}(x)=0, x \neq 0, x \in \mathbb{Z}$, it follows from Definition 6 that

$$
\Phi_{n}(x)=0, \quad|x|>n, \quad x \in \mathbb{Z},
$$

which gives

$$
\sum_{x=-\infty}^{\infty}|x|^{2 r}\left\|\Phi_{n}(x)\right\|^{2}=\sum_{x=-n}^{n}|x|^{2 r}\left\|\Phi_{n}(x)\right\|^{2}<\infty,
$$

which together with (27) implies $\Phi_{n} \in \operatorname{dom} X^{r}$. By using (28), we immediately get

$$
\begin{aligned}
\left\langle\Phi_{n}, X^{r} \Phi_{n}\right\rangle_{l^{2}} & =\sum_{x=-\infty}^{\infty}\left\langle\Phi_{n}(x), x^{r} \Phi_{n}(x)\right\rangle \\
& =\sum_{x=-\infty}^{\infty} x^{r}\left\|\Phi_{n}(x)\right\|^{2} .
\end{aligned}
$$

This competes the proof.

It is easy to verify that $\left\{e^{i t} R_{n}+e^{-i t} L_{n} \mid t \in \mathbb{R}, n \geq 0\right\}$ makes a commutative family of unitary operators on $\mathscr{H}$. Here, by convention, $\mathbb{R}$ denotes the set of all real numbers. In the following, for $n \geq 0$, we define operator-valued function $V_{n}(t)$ as

$$
V_{n}(t)=e^{i t} R_{n}+e^{-i t} L_{n}, \quad t \in \mathbb{R}
$$

which is continuous and has continuous derivatives up to order $r$ with the operator norm for any positive integer $r$.

Proposition 15. Let $n \geq 0$ and $r \geq 1$ be integers. Then the rth derivative of the operator-valued function $V_{n}(t)$ satisfies

$$
\frac{d^{r} V_{n}(t)}{d t^{r}}=i^{r}\left[V_{n}(0)\right]^{r} V_{n}(t)
$$

where $i$ denotes the imaginary unit. 
Proof. A direct calculation gives

$$
\frac{d V_{n}(t)}{d t}=\left(e^{i t} R_{n}+e^{-i t} L_{n}\right)^{\prime}=i\left(e^{i t} R_{n}-e^{-i t} L_{n}\right) .
$$

On the other hand, by using Lemma 5, we find

$$
\begin{aligned}
V_{n}(0) V_{n}(t) & =\left(R_{n}+L_{n}\right)\left(e^{i t} R_{n}+e^{-i t} L_{n}\right) \\
& =e^{i t} R_{n}-e^{-i t} L_{n} .
\end{aligned}
$$

Thus $d V_{n}(t) / d t=i V_{n}(0) V_{n}(t)$. By induction, formula (44) follows.

Proposition 16. Let $n \geq 0$ and $r \geq 1$ be integers. Then it holds that

$$
\frac{d^{r}}{d t^{r}}\left[\prod_{k=0}^{n} V_{k}(t)\right]=\left[i \sum_{k=0}^{n} V_{k}(0)\right]^{r} \prod_{k=0}^{n} V_{k}(t) .
$$

Proof. By using Proposition 15 and induction, we can get the desired result easily.

As an immediate consequence of Propositions 15 and 16, we have the next proposition.

Proposition 17. Let $\varphi \in \mathscr{H}$, and $n \geq 0$. Define function $f$ : $[0,2 \pi] \rightarrow \mathscr{H}$ as

$$
f(t)=\left[\prod_{k=0}^{n} V_{k}(t)\right] \varphi, \quad t \in[0,2 \pi] .
$$

Then, for any integer $r \geq 1, f$ is a continuous function on $[0,2 \pi]$ that has continuous derivatives up to order $r$, and moreover

$$
f^{(r)}(t)=\left[i \sum_{k=0}^{n} V_{k}(0)\right]^{r} f(t), \quad t \in[0,2 \pi] .
$$

Proposition 18. Let $\varphi \in \mathscr{H}$, and $n \geq 0$. Let $f$ be the same as in Proposition 17. Then $f^{(r)} \in L^{2}(\mathbb{S}, \mathscr{H})$ for all integers $r \geq 0$.

Proof. We first fix some notation. Let $\Gamma_{n]}=\{\sigma \in \Gamma \mid \max \sigma \leq$ $n\}$. For $\sigma \in \Gamma_{n]}$, we put

$$
R_{\sigma}=\prod_{k \in \sigma} R_{k}
$$

where $R_{\sigma}=I$ when $\sigma=\emptyset$. Similarly, we use $L_{\sigma}$ for $\sigma \in \Gamma_{n]}$.

Now, by a direct calculation, we can get

$$
f(t)=\sum_{\sigma \in \Gamma_{n]}} e^{i(2 \# \sigma-n-1) t} R_{\sigma} L_{\sigma^{c}} \varphi, \quad t \in[0,2 \pi],
$$

where $\sigma^{c}=\{0,1, \ldots, n\} \backslash \sigma$. Clearly, for each $\sigma \in \Gamma_{n]}$, the function

$$
t \longmapsto e^{i(2 \# \sigma-n-1) t} R_{\sigma} L_{\sigma^{c}} \varphi
$$

belongs to $L^{2}(\mathbb{S}, \mathscr{H})$, which implies $f \in L^{2}(\mathbb{S}, \mathscr{H})$. Using formula (49) and formula (51), we can similarly show that $f^{(r)} \in L^{2}(\mathbb{S}, \mathscr{H})$ holds for all integers $r \geq 0$.
Recall that $\partial_{k}$ and $\partial_{k}^{*}$ denote the annihilation and creation operators, respectively, which are members of quantum Bernoulli noise. The next theorem then offers a formula that links the moments of the QBN-based walk's probability distributions directly with the annihilation and creation operators.

Theorem 19. Let $\left(\Phi_{n}\right)_{n \geq 0} \subset l^{2}(\mathbb{Z}, \mathscr{H})$ be the state sequence of the $Q B N$-based walk. Suppose the initial state $\Phi_{0}$ takes the following form:

$$
\Phi_{0}(x)= \begin{cases}\varphi, & x=0 \\ 0, & x \neq 0, x \in \mathbb{Z},\end{cases}
$$

where $\varphi \in \mathscr{H}$ with $\|\varphi\|=1$. Then, the rth moment $\left\langle\Phi_{n}\right.$, $\left.X^{r} \Phi_{n}\right\rangle_{l^{2}}$ of the walk's probability distribution at time $n \geq 1$ satisfies

$$
\left\langle\Phi_{n}, X^{r} \Phi_{n}\right\rangle_{l^{2}}=\left\langle\varphi,\left[\sum_{k=0}^{n-1}\left(\partial_{k}^{*}+\partial_{k}\right)\right]^{r} \varphi\right\rangle,
$$

where $r \geq 1$ is a positive integer.

Proof. By Theorem 14, we know $\Phi_{n} \in \operatorname{dom} X^{r}$ for all $n \geq 0$ and $r \geq 1$. Let $f_{n}=\mathrm{F} \Phi_{n}$ for $n \geq 0$. Then $f_{0}(t)=\varphi, t \in[0,2 \pi]$, and by Theorems 12 and 11,

$$
f_{n}(t)=\left[\prod_{k=0}^{n-1} V_{k}(t)\right] \varphi, \quad t \in[0,2 \pi], n \geq 1 .
$$

Now let $n, r \geq 1$. Then, by Remark 10 , we have

$$
\begin{aligned}
\left\langle\Phi_{n}, X^{r} \Phi_{n}\right\rangle_{l^{2}} & =(-i)^{r}\left\langle f_{n}, f_{n}^{(r)}\right\rangle_{L^{2}} \\
& =\frac{(-i)^{r}}{2 \pi} \int_{0}^{2 \pi}\left\langle f_{n}(t), f_{n}^{(r)}(t)\right\rangle d t,
\end{aligned}
$$

which together with Proposition 17 gives

$$
\begin{aligned}
& \left\langle\Phi_{n}, X^{r} \Phi_{n}\right\rangle_{l^{2}} \\
& \quad=\frac{1}{2 \pi} \int_{0}^{2 \pi}\left\langle f_{n}(t),\left[\sum_{k=0}^{n} V_{k}(0)\right]^{r} f_{n}(t)\right\rangle d t .
\end{aligned}
$$

On the other hand, by using the commutativity of the unitary operator family $\left\{V_{k}(t)\right\}$ as well as (55), we have

$$
\begin{aligned}
& \left\langle f_{n}(t),\left[\sum_{k=0}^{n} V_{k}(0)\right]^{r} f_{n}(t)\right\rangle \\
& =\left\langle\left[\prod_{k=0}^{n-1} V_{k}(t)\right] \varphi,\left[\sum_{k=0}^{n} V_{k}(0)\right]^{r}\left[\prod_{k=0}^{n-1} V_{k}(t)\right] \varphi\right\rangle \\
& =\left\langle\left[\prod_{k=0}^{n-1} V_{k}(t)\right] \varphi,\left[\prod_{k=0}^{n-1} V_{k}(t)\right]\left[\sum_{k=0}^{n} V_{k}(0)\right]^{r} \varphi\right\rangle \\
& =\left\langle\varphi,\left[\sum_{k=0}^{n} V_{k}(0)\right]^{r} \varphi\right\rangle,
\end{aligned}
$$


where $t \in[0,2 \pi]$. Thus

$$
\begin{aligned}
\left\langle\Phi_{n}, X^{r} \Phi_{n}\right\rangle_{l^{2}} & =\frac{1}{2 \pi} \int_{0}^{2 \pi}\left\langle\varphi,\left[\sum_{k=0}^{n} V_{k}(0)\right]^{r} \varphi\right\rangle d t \\
& =\left\langle\varphi,\left[\sum_{k=0}^{n-1} V_{k}(0)\right]^{r} \varphi\right\rangle
\end{aligned}
$$

which, together with the fact that $V_{k}(0)=\partial_{k}^{*}+\partial_{k}, k \geq 0$, yields the desired formula.

\section{Quantum Central Limit Theorem}

Quantum central limit theorems are quantum analogs of the classical central limit theorem, which deal with observables from a quantum probability point of view. In the present section, we use the results obtained in the previous section to prove a quantum central limit theorem for quantum Bernoulli noise itself.

In what follows, we denote by $\mathscr{B}(\mathbb{R})$ the Borel $\sigma$-filed over the real line $\mathbb{R}$. For a Borel set $A \in \mathscr{B}(\mathbb{R})$, we sue $\mathbf{1}_{A}$ to mean its indicator as usual.

Recall that $\mathscr{H}$ denotes the space of square integrable Bernoulli functionals, which serves as the coin space of the QBN-based walk. Now consider the following observables:

$$
\Xi_{k}=\partial_{k}^{*}+\partial_{k}, \quad k \geq 0
$$

where $\partial_{k}$ and $\partial_{k}^{*}$ are the annihilation and creation operators on $\mathscr{H}$, which are members of quantum Bernoulli noise. Let $\varphi \in \mathscr{H}$ be a unit vector. Then, by the well-known von Neumann's spectral theorem [27], there exists a sequence $\left(\mu_{n}^{(\varphi)}\right)_{n \geq 1}$ of Borel probability measures on the real line $\mathbb{R}$ such that

$$
\int_{\mathbb{R}} t^{r} d \mu_{n}^{(\varphi)}(t)=\left\langle\varphi,\left[\frac{1}{\sqrt{n}} \sum_{k=0}^{n-1} \Xi_{k}\right]^{r} \varphi\right\rangle, \quad r \geq 1,
$$

where $\langle\cdot, \cdot\rangle$ denotes the inner product of the space $\mathscr{H}$.

Theorem 20. Let $\left(\mu_{n}^{(\varphi)}\right)_{n \geq 1}$ be the probability measure sequence described in (61). Suppose the unit vector $\varphi \in \mathscr{H}$ takes the form $\varphi=\alpha Z_{\emptyset}+\beta Z_{0}$, where $\alpha, \beta \in \mathbb{C}$ with $|\alpha|^{2}+|\beta|^{2}=1$. Then, for each $n \geq 1$, the probability measure $\mu_{n}^{(\varphi)}$ has the following representation:

$$
\begin{aligned}
& \mu_{n}^{(\varphi)}(A)=\frac{1}{2^{n}} \sum_{j=0}^{n} \mathbf{1}_{A}\left(\frac{2 j-n}{\sqrt{n}}\right) \\
& \cdot\left[\left(\begin{array}{c}
n-1 \\
j
\end{array}\right)|\alpha-\beta|^{2}+\left(\begin{array}{c}
n-1 \\
j-1
\end{array}\right)|\alpha+\beta|^{2}\right], \\
& A \in \mathscr{B}(\mathbb{R}),
\end{aligned}
$$

where $\left(\begin{array}{c}n-1 \\ -1\end{array}\right)=\left(\begin{array}{c}n-1 \\ n\end{array}\right)=0$ and $\left(\begin{array}{l}0 \\ 0\end{array}\right)=1$.

Proof. Consider the QBN-based walk with the initial state $\Phi_{0}$ given by

$$
\Phi_{0}(x)= \begin{cases}\varphi, & x=0 \\ 0, & x \neq 0, \quad x \in \mathbb{Z}\end{cases}
$$

Let $\Phi_{n}$ be the state of the walk at time $n$ and $Y_{n}$ a random variable with the following probability distribution:

$$
P\left\{Y_{n}=x\right\}=\left\|\Phi_{n}(x)\right\|^{2}, \quad x \in \mathbb{Z} .
$$

Then it follows from the proof of Theorem 4.5 of [13] that

$$
P\left\{Y_{n}=x\right\}=\left\{\begin{array}{l}
\frac{1}{2^{n}}\left[\left(\begin{array}{c}
n-1 \\
j
\end{array}\right)|\alpha-\beta|^{2}+\left(\begin{array}{c}
n-1 \\
j-1
\end{array}\right)|\alpha+\beta|^{2}\right], \quad x=2 j-n, 0 \leq j \leq n \\
0, \quad \text { otherwise. }
\end{array}\right.
$$

On the other hand, by using (61) as well as Theorems 19 and 14 , we can get

$$
\begin{aligned}
\int_{\mathbb{R}} t^{r} d \mu_{n}^{(\varphi)}(t) & =\left\langle\Phi_{n},\left(\frac{X}{\sqrt{n}}\right)^{r} \Phi_{n}\right\rangle_{l^{2}} \\
& =\sum_{x=-\infty}^{\infty}\left(\frac{x}{\sqrt{n}}\right)^{r}\left\|\Phi_{n}(x)\right\|^{2}, \quad r \geq 1,
\end{aligned}
$$

which, together with the fact that both $\mu_{n}^{(\varphi)}$ and the distribution of $Y_{n} / \sqrt{n}$ have compact supports, yields

$$
\int_{\mathbb{R}} e^{i \theta t} d \mu_{n}^{(\varphi)}(t)=\sum_{r=0}^{\infty} \frac{(i \theta)^{r}}{r !} \int_{\mathbb{R}} t^{r} d \mu_{n}^{(\varphi)}(t)
$$

$$
\begin{aligned}
& =\sum_{r=0}^{\infty} \frac{(i \theta)^{r}}{r !} \sum_{x=-\infty}^{\infty}\left(\frac{x}{\sqrt{n}}\right)^{r}\left\|\Phi_{n}(x)\right\|^{2} \\
& =\sum_{x=-\infty}^{\infty} e^{i \theta(x / \sqrt{n})}\left\|\Phi_{n}(x)\right\|^{2} \\
& =E e^{i \theta\left(Y_{n} / \sqrt{n}\right)}, \quad \theta \in \mathbb{R},
\end{aligned}
$$

which implies that $\mu_{n}^{(\varphi)}$ is exactly the same as the distribution of $Y_{n} / \sqrt{n}$. Thus, by (65), we get (62). 
Theorem 21. Let $\left(\mu_{n}^{(\varphi)}\right)_{n \geq 1}$ be the probability measure sequence described in (61). Suppose the unit vector $\varphi \in \mathscr{H}$ takes the form $\varphi=\alpha Z_{\emptyset}+\beta Z_{0}$, where $\alpha, \beta \in \mathbb{C}$ with $|\alpha|^{2}+|\beta|^{2}=1$. Then

$$
\mu_{n}^{(\varphi)} \Longrightarrow N(0,1) \quad \text { as } n \longrightarrow \infty
$$

namely, the probability measure sequence $\left(\mu_{n}^{(\varphi)}\right)_{n \geq 1}$ converges weakly to the standard Gaussian distribution $N(0,1)$ on the real line $\mathbb{R}$.

Proof. Again consider the QBN-based walk with the initial state $\Phi_{0}$ given by

$$
\Phi_{0}(x)= \begin{cases}\varphi, & x=0 \\ 0, & x \neq 0, \quad x \in \mathbb{Z} .\end{cases}
$$

For each $n \geq 1$, let $\Phi_{n}$ be the state of the walk at time $n$ and $Y_{n}$ a random variable with the following probability distribution:

$$
P\left\{Y_{n}=x\right\}=\left\|\Phi_{n}(x)\right\|^{2}, \quad x \in \mathbb{Z} .
$$

Then, by Theorem 4.5 of [13], $Y_{n} / \sqrt{n} \Rightarrow N(0,1)$, which, together with (67) in the proof of Theorem 20, yields

$$
\lim _{n \rightarrow \infty} \int_{\mathbb{R}} e^{i \theta t} d \mu_{n}^{(\varphi)}(t)=\lim _{n \rightarrow \infty} E e^{i \theta\left(Y_{n} / \sqrt{n}\right)}=e^{-\theta^{2} / 2}
$$

$$
\theta \in \mathbb{R}
$$

which then implies that $\mu_{n}^{(\varphi)} \Rightarrow N(0,1)$ as $n \rightarrow \infty$.

Remark 22. As can be seen, observables $\Xi_{k}=\partial_{k}^{*}+\partial_{k}, k \geq 0$, actually play the role of quantum bias in the construction of the QBN-based walk. Theorem 21 then establishes a quantum central limit theorem for these observables.

\section{Conclusion Remarks}

In the final section, we would like to make some further remarks about the QBN-based walk.

As is known, the QBN-based walk belongs to the category of discrete-time unitary quantum walks on the line. However, it is still different from a usual discrete-time unitary quantum walk on the line. In fact, the QBN-based walk is of the following form:

$$
\widehat{\Phi}_{n+1}(t)=\widehat{U}_{n} \widehat{\Phi}_{n}(t),
$$

where $\left\langle x \mid \Phi_{n}\right\rangle$ belongs to a Hilbert coin space, $\widehat{\Phi}_{n}(t)$ is its Fourier transform, and

$$
\widehat{U}_{n}=\frac{1}{2}\left[e^{i t}\left(B_{n}+I\right)+e^{-i t}\left(B_{n}-I\right)\right],
$$

where $B_{n}$ is a self-adjoint unitary operator acting on the coin space. However, a usual discrete-time unitary quantum walk on the line (take the one with a 2-dimensional coin space as example) reads

$$
\widehat{\Phi}_{n+1}(t)=\widehat{S} C_{n} \widehat{\Phi}_{n}(t)
$$

where $C_{n}$, called the coin operator, acts the coin space, and

$$
\widehat{S}=\left(\begin{array}{cc}
e^{i t} & 0 \\
0 & e^{-i t}
\end{array}\right)
$$

is known as the spin-dependent shift operator.

As shown in Theorem 21, the limit probability distribution of the QBN-based walk for a localized initial state can lead to a quantum central limit theorem for observables $\partial_{k}^{*}+\partial_{k}, k \geq 0$. However, only for some special localized initial states, have we obtained the walk's limit probability distributions. It is still unclear whether or not the walk has a limit probability distribution for a general localized initial state. On the other hand, as its name suggests, the QBNbased walk might be viewed as such a quantum walk in an open environment that its evolution will be affected by the effects of environment described by quantum Bernoulli noise. In other words, decoherence might happen in the evolution of the walk. Thus we conjecture that, for a general localized initial state, the QBN-based walk might still have the same limit probability distribution as the classical random walk.

The Bernoulli-type random variables $\left(Z_{n}\right)_{n \in \mathbb{N}}$ described in Section 2 play an important role in understanding the structure of the coin space $\mathscr{H}$ of the QBN-based walk. However, those parameters $p_{n}, q_{n}$ in their distributions actually have nothing to do with the properties of the walk although an explicit $n$-dependence is indicated by $p_{n}$ and $q_{n}$. More precisely, the properties of the QBN-based walk are independent of the choice of those parameters $p_{n}, q_{n}$ in the distributions of random variables $\left(Z_{n}\right)_{n \in \mathbb{N}}$, and indicating explicitly the $n$-dependence of $p_{n}$ and $q_{n}$ is only for the sake of generality. In fact, one can weaken the conditions on $\left(Z_{n}\right)_{n \in \mathbb{N}}$ without invalidating the main theorems presented in this paper. For instance, one can take $\left(Z_{n}\right)_{n \in \mathbb{N}}$ such that it is a "discrete-time (correlated) noise" with the chaotic representation property (see $[25,28])$.

\section{Conflicts of Interest}

The authors declare that they have no conflicts of interest.

\section{Acknowledgments}

This work is supported by National Natural Science Foundation of China (Grant no. 11461061).

\section{References}

[1] J. Kempe, "Quantum random walks: An introductory overview," Contemporary Physiscs, vol. 44, no. 4, pp. 307-327, 2003.

[2] N. Konno, "Quantum walks," in Quantum Potential Theory, vol. 1954 of Lecture Notes in Math, pp. 309-452, Springer, Berlin, Germany, 2008.

[3] S. E. Venegas-Andraca, "Quantum walks: a comprehensive review," Quantum Information Processing, vol. 11, no. 5, pp. 10151106, 2012.

[4] G. Grimmett, S. Janson, and P. F. Scudo, "Weak limits for quantum random walks," Physical Review E: Statistical, Nonlinear, 
and Soft Matter Physics, vol. 69, no. 2, Article ID 026119, pp. 126119, 2004.

[5] Y. Higuchi, N. Konno, I. Sato, and E. Segawa, "Spectral and asymptotic properties of Grover walks on crystal lattices," Journal of Functional Analysis, vol. 267, no. 11, pp. 4197-4235, 2014.

[6] T. Machida, "Realization of the probability laws in the quantum central limit theorems by a quantum walk," Quantum Information \& Computation, vol. 13, no. 5-6, pp. 430-438, 2013.

[7] K. Matsue, O. Ogurisu, and E. Segawa, "Quantum walks on simplicial complexes," Quantum Information Processing, vol. 15, no. 5, pp. 1865-1896, 2016.

[8] E. Segawa and A. Suzuki, "Generator of an abstract quantum walk," Quantum Studies. Mathematics and Foundations, vol. 3, no. 1, pp. 11-30, 2016.

[9] A. Suzuki, "Asymptotic velocity of a position-dependent quantum walk," Quantum Information Processing, vol. 15, no. 1, pp. 103-119, 2016.

[10] N. Konno, "A new type of limit theorems for the onedimensional quantum random walk," Journal of the Mathematical Society of Japan, vol. 57, no. 4, pp. 1179-1195, 2005.

[11] N. Konno, "Limit theorem for continuous-time quantum walk on the line," Physical Review E: Statistical, Nonlinear, and Soft Matter Physics, vol. 72, no. 2, Article ID 026113, 2005.

[12] Z. Y. Huang, "Quantum white noises-white noise approach to quantum stochastic calculus," Nagoya Mathematical Journal, vol. 129, pp. 23-42, 1993.

[13] C. Wang and X. Ye, "Quantum walk in terms of quantum Bernoulli noises," Quantum Information Processing, vol. 15, no. 5, pp. 1897-1908, 2016.

[14] C. D. Cushen and R. L. Hudson, "A quantum-mechanical central limit theorem," Journal of Applied Probability, vol. 8, pp. 454-469, 1971.

[15] J. Quaegebeur, "A noncommutative central limit theorem for CCR-algebras," Journal of Functional Analysis, vol. 57, no. 1, pp. $1-20,1984$.

[16] N. Giri and W. von Waldenfels, "An algebraic version of the central limit theorem," Zeitschrift für Wahrscheinlichkeitstheorie und Verwandte Gebiete, vol. 42, no. 2, pp. 129-134, 1978.

[17] D. Voiculescu, "Lectures on free probability theory," in Lectures on probability theory and statistics (Saint-Flour, 1998), vol. 1738 of Lecture Notes in Math, pp. 279-349, Springer, Berlin, Germany, 2000.

[18] D. Goderis, A. Verbeure, and P. Vets, "Noncommutative central limits," Probability Theory and Related Fields, vol. 82, no. 4, pp. 527-544, 1989.

[19] G. Kuperberg, "A tracial quantum central limit theorem," Transactions of the American Mathematical Society, vol. 357, no. 2, pp. 459-471, 2005.

[20] R. Lenczewski, "Quantum central limit theorems," in Symmetries in science, VIII (Bregenz, 1994), pp. 299-314, Plenum, New York, NY, USA, 1995.

[21] Y. G. Lu, "On the interacting free Fock space and the deformed Wigner law," Nagoya Mathematical Journal, vol. 145, pp. 1-28, 1997.

[22] N. Muraki, "Noncommutative Brownian motion in monotone Fock space," Communications in Mathematical Physics, vol. 183, no. 3, pp. 557-570, 1997.

[23] C. Wang, H. Chai, and Y. Lu, "Discrete-time quantum Bernoulli noises," Journal of Mathematical Physics, vol. 51, no. 5, Article ID 009006JMP, 2010.
[24] C. Wang and J. Chen, "Quantum Markov semigroups constructed from quantum Bernoulli noises," Journal of Mathematical Physics, vol. 57, no. 2, Article ID 023502, 2016.

[25] N. Privault, "Stochastic analysis of Bernoulli processes," Probability Surveys, vol. 5, pp. 435-483, 2008.

[26] J. Diestel and J. J. Uhl, Vector Measures, vol. 15 of American Mathematical Society, Mathematical Surveys and Monographs, Providence, RI, USA, 1977.

[27] K. R. Parthasarathy, An Introduction to Quantum Stochastic Calculus, vol. 85 of Monographs in Mathematics, Birkhäuser, Basel, Switzerland, 1992.

[28] C. S. Wang, Y. C. Lu, and H. F. Chai, "An alternative approach to Privault's discrete-time chaotic calculus," Journal of Mathematical Analysis and Applications, vol. 373, no. 2, pp. 643-654, 2011. 


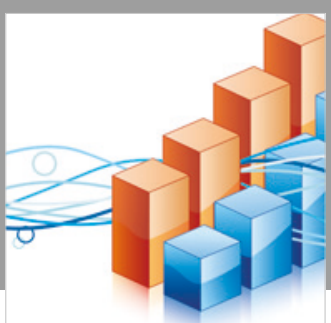

Advances in

Operations Research

\section{-n-m}
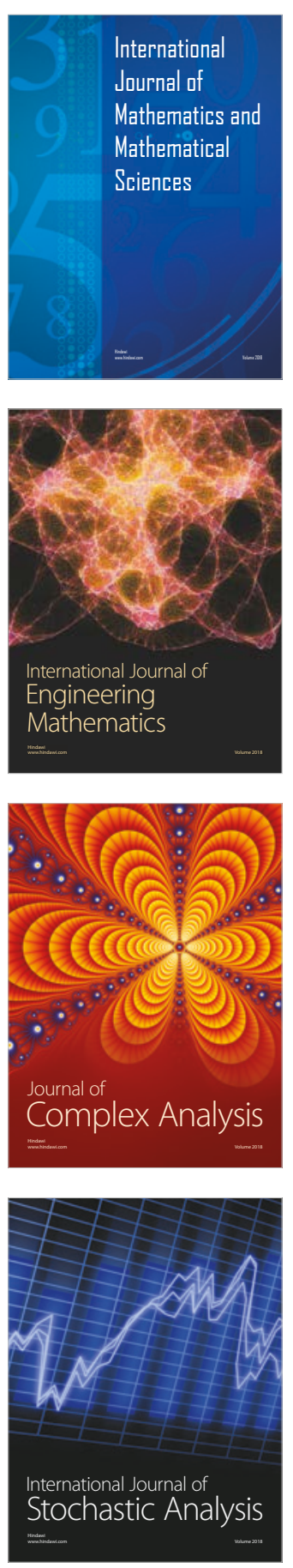
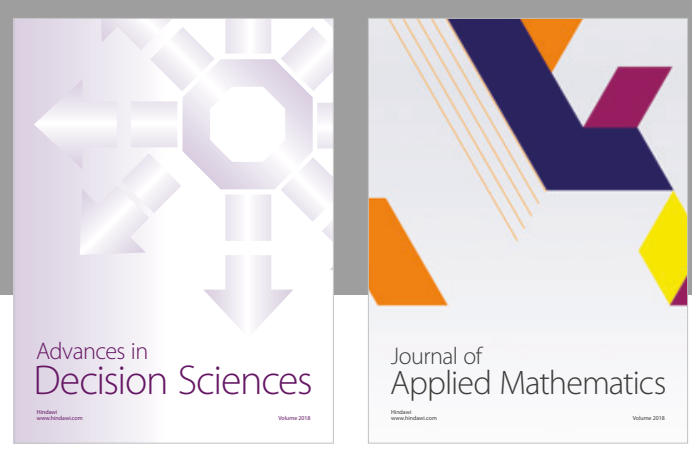

Journal of

Applied Mathematics
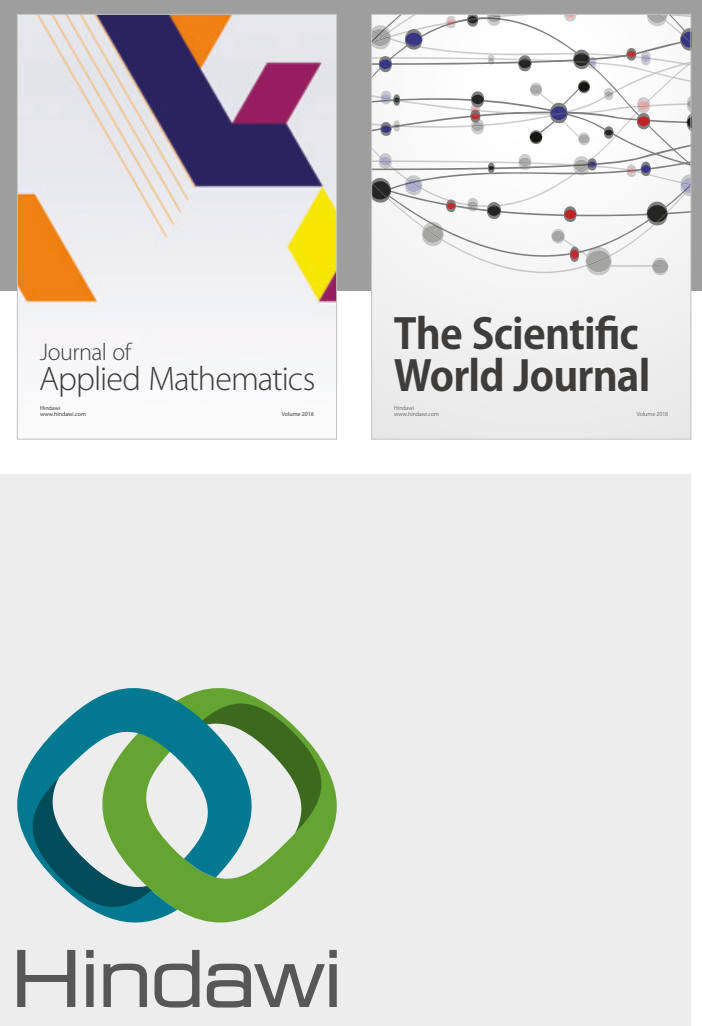

Submit your manuscripts at

www.hindawi.com

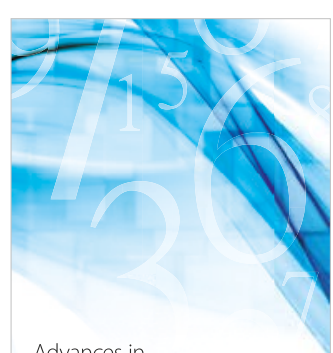

Advances in
Numerical Analysis
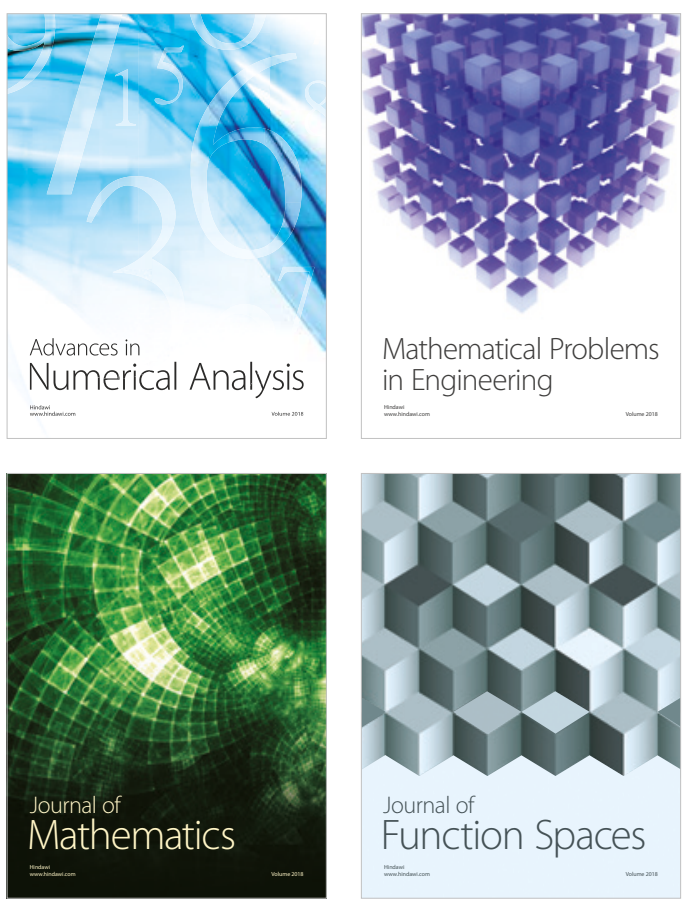

Mathematical Problems in Engineering

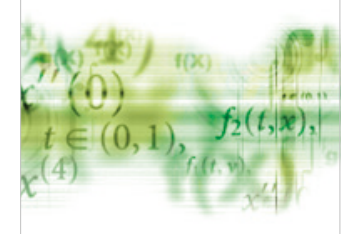

International Journal of

Differential Equations

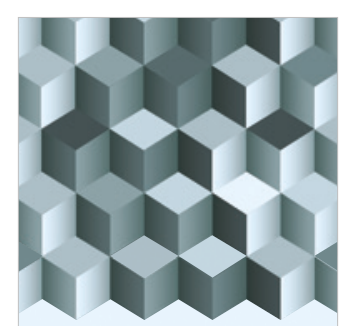

Journal of

Function Spaces

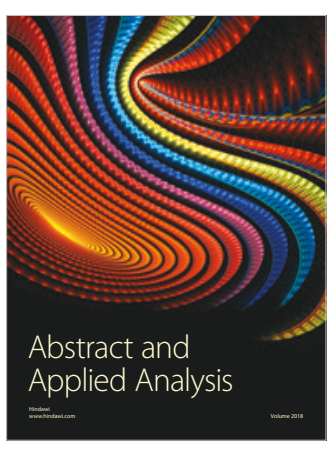

The Scientific

World Journal

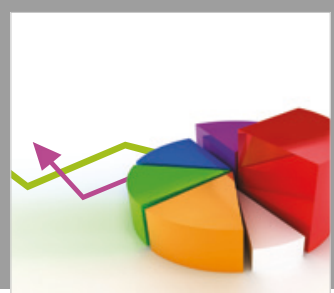

Journal of

Probability and Statistics
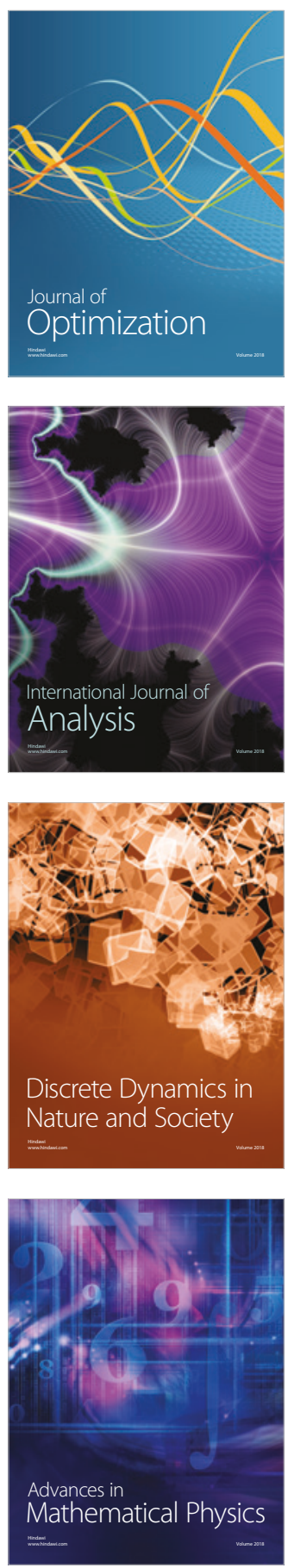